Diabetologe 2011 - 7:559-560

DOI 10.1007/s11428-011-0732-x

Online publiziert: 1. Dezember 2011

(c) Springer-Verlag 2011

\author{
N.C. Schloot ${ }^{1,2,3}$ \\ ${ }^{1}$ Institut für Klinische Diabetologie, Deutsches Diabetes-Zentrum, \\ Leibniz-Zentrum für Diabetesforschung, Heinrich-Heine-Universität, Düsseldorf \\ ${ }^{2}$ Klinik für Stoffwechselkrankheiten, Universitätsklinikum Düsseldorf \\ ${ }^{3}$ Lilly Deutschland, Bad Homburg
}

\title{
Auf dem Weg zur Prävention des Typ-1-Diabetes
}

sulinproduzierenden $\beta$-Zellen verantwortlich gemacht. Hier spielen autoreaktive T-Helfer und T-zytotoxische Zellen mit ihren Immunmediatoren sowie dysfunktionelle regulatorische T-Zellen zusammen mit den antigenpräsentierenden Zellen eine wichtige Rolle. Wiederholt gab und gibt es Versuche, den Krankheitsprozess nach Manifestation des Typ-1-Diabetes (Tertiärprävention), bei inselantikörperpositiven Risikopersonen (Sekundärprävention) oder autoantikörpernegativen, genetisch definierten Risikopersonen (Primärprävention) zu verlangsamen oder bestenfalls die Personen zu heilen. Diese Phaseneinteilung ist an die einzelnen Stadien des (Prä-)Typ-1-Diabetes adaptiert und steht im Gegensatz zu anderen Erkrankungen, bei denen sonst versucht wird, mit der Primärprävention die Erkrankung zu verhindern, mit der Sekundärprävention Komplikationen zu verhindern und mit der Tertiärprävention das Wiederauftreten von Komplikationen zu verhindern.

In der Tertiärprävention des Typ-1Diabetes gab es in den 1980er Jahren mit dem Immunmodulator Ciclosporin A die ersten Teilerfolge, bei denen der progrediente Verlust der endogenen Insulinproduktion vorübergehend aufgehalten werden konnte. Wie bei vielen anderen immunologischen Therapieansätzen waren jedoch die Nebenwirkungen in Anbetracht der durchschnittlich guten Prognose bei Typ-1-Diabetes nicht akzeptabel. Die Sekundärprävention wurde in den 1990er Jahren favorisiert; viele Tausende Risikopersonen wurden untersucht, um inselautoantikörperpositive Studienteilnehmer zu identifizieren. Leider zeigten weder das amerikanische Diabetes Prevention Trial mit Insulin (DPT-1) noch das europäische European Nicotinamide Diabetes Intervention Trial (ENDIT) oder die Deutsche Nikotinamid-InterventionStudie (DENIS) Erfolg. Nach einer relativen Pause etablierten sich neue Ansätze, häufig mit Fokus auf die Tertiärprävention; diese neueren Studien werden in dieser Ausgabe von Der Diabetologe zusammengefasst und kritisch beurteilt.

Die Tertiärprävention fokussiert auf die zeitnahe Intervention nach Diabetesdiagnose. Bei bereits lange Jahre bestehendem Typ-1-Diabetes ist die Inselzell- oder Pankreastransplantation eine kurative Intervention - allerdings ist hier immer eine immunsuppressive Begleitmedikation nötig. Der Therapieerfolg, insbesondere bei der Inselzelltransplantation, ist meist nicht langfristig garantiert, sodass diese Therapieoptionen derzeit nicht für ansonsten gesunde Typ-1-Diabetes-Patienten angeboten werden.

Relativ neu sind Therapieversuche, bei denen eine autologe Stammzelltransplantation nach Konditionierung und Knochenmarkablation vorgenommen wird. Die hierzu verwendeten Protokolle sind aufwendig und haben schwerwiegende Nebenwirkungen entsprechend einer Chemotherapie, konnten aber zeigen, dass auch nach Manifestation des Typnetisch wird die zelluläre Immunreaktion für die selektive Destruktion der in- 
1-Diabetes eine lang anhaltende Remission ohne exogenes Insulin erreichbar ist. Möglichkeiten zur verbesserten Regeneration der $\beta$-Zellen oder die Umprogrammierung von Nicht- $\beta$-Zellen in $\beta$ Zellen werden derzeit in der Stammzellforschung intensiv studiert. Es bleibt abzuwarten, wann hier Therapieversuche am Menschen in vivo begonnen werden können.

- Realistisches Ziel ist die

Verlangsamung des Verlusts

der restlichen endogenen

Insulinproduktion.

Wahrscheinlich führt nicht eine Monotherapie, sondern die Kombinationstherapie des Typ-1-Diabetes mittelfristig zum Erfolg. Diese Strategie wird bereits bei anderen immunvermittelten Erkrankungen wie rheumatoider Arthritis und multipler Sklerose angewandt, sodass man sich fragt, wann die Kombinationstherapie auch bei Typ-1-Diabetes in größer angelegten Studien getestet werden kann.

Patienten mit Typ-1-Diabetes oder Risikopersonen sollten ausführlich aufgeklärt werden, wenn sie für eine Studienteilnahme infrage kommen. Die Teilnahme an solchen Studien ist allerdings der einzige Weg, Fortschritte zum langfristigen Erhalt der $\beta$-Zell-Funktion zu erreichen.

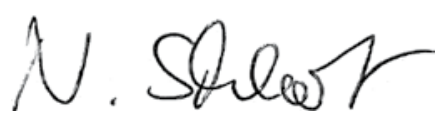

N. Schloot

\section{Korrespondenzadresse}

PD Dr. N.C. Schloot

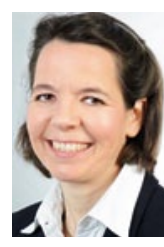

Institut für Klinische Diabetologie, Deutsches Diabetes-Zentrum, Leibniz-Zentrum für Diabetesforschung, Heinrich-Heine-Universität Auf'm Hennekamp 65, 40225 Düsseldorf schloot@ddz.uni-duesseldorf.de

\section{Galenus-von-}

Pergamon-Preis 2011

\section{Springer Medizin zeichnet exzellente Forschung und ehrenamtliches Engagement aus}

Auch in diesem Jahr hat Springer Medizin den von der Ärzte Zeitung Verlags $\mathrm{GmbH}$ gestifteten Galenus-von-Pergamon-Preis vergeben und würdigt damit exzellente Forschung in Deutschland. 12 unabhängige Experten haben im Oktober 2011 über die Preisträger in den Kategorien Primary Care, Specialist Care und Grundlagenforschung entschieden. Als Schirmherrin des Galenus-von-PergamonPreises lobte die Bundesministerin für Bildung und Forschung, Frau Professor Dr. Annette Schavan, die herausragenden Leistungen aller Forscher-Teams.

Primary Care

Der Preis in der Kategorie Primary Care würdigt ein Medikament, das bei einer breiten Patientengruppe eingesetzt wird. In diesem Jahr hat Amgen/GlaxoSmithKline (GSK) den Preis für Prolia ${ }^{\circledast}$ erhalten. Mit Prolia $^{\circledR}$ steht erstmals ein monoklonaler Antikörper zur gezielten Osteoporosetherapie zur Verfügung. Der Antiköper hemmt die Knochenresorption und schützt somit Frauen in der Postmenopause und Männer mit Prostatakrebs vor Frakturen.

\section{Specialist Care}

Der Preis in der Kategorie Specialist Care zeichnet ein Medikament aus, das zur Behandlung seltener Erkrankungen verwendet wird. In diesem Jahr ist Amgen der Gewinner für Nplate ${ }^{\circledR}$, das erste zugelassene Medikament zur Stimulierung der Thrombozytenbildung. Es ist indiziert zur Behandlung von Erwachsenen mit einer chronischen Immun-(idiopathischen)thrombozytopenischen Purpura (ITP), die auf andere Therapien nicht ansprechen.
Die Preisträger in diesen beiden Kategorien erhielten jeweils eine Medaille und eine Urkunde.

\section{Grundlagenforschung}

In dieser Kategorie wird ein Bewerber prämiert, der eine herausragende wissenschaftliche Arbeit in der pharmakologischen Grundlagenforschung eingereicht hat. Die Auszeichnung ging an Professor Wolfgang Kühn aus Freiburg. Er und sein Team haben sich in der Forschung um die Entschlüsselung molekularer Mechanismen der autosomal dominanten polyzystischen Nierenerkrankung (ADPKD) verdient gemacht. Der Preisträger erhält zur Medaille und Urkunde zusätzlich ein Preisgeld in Höhe von 10.000 Euro.

CharityAward 2011

Mit dem im Jahr 2009 erstmals gestifteten CharityAward zeichnet Springer Medizin jährlich Menschen und Organisationen aus, die sich ehrenamtlich um behinderte, kranke und hilfsbedürftige Menschen verdient gemacht haben. Wer Gewinner wird, entscheiden die Leser der Medien von Springer Medizin. Preisträgerin 2011 ist die in Peru geborene Ärztin Jenny De la Torre: Sie wird für ihren inzwischen 16-jährigen Einsatz für Obdachlose in Berlin-Mitte ausgezeichnet. Im Rahmen dieses Engagements hat sie eine Stiftung gegründet und das Berliner "Gesundheitszentrum für Obdachlose" aufgebaut. Der Award besteht aus einem Medienpaket in einem Wert von 100.000 Euro und einem Barscheck über 50.000 Euro. Schirmherr des Preises ist Bundesgesundheitsminister Daniel Bahr. 